\title{
Report on the Second MPEX User Research Forum
}

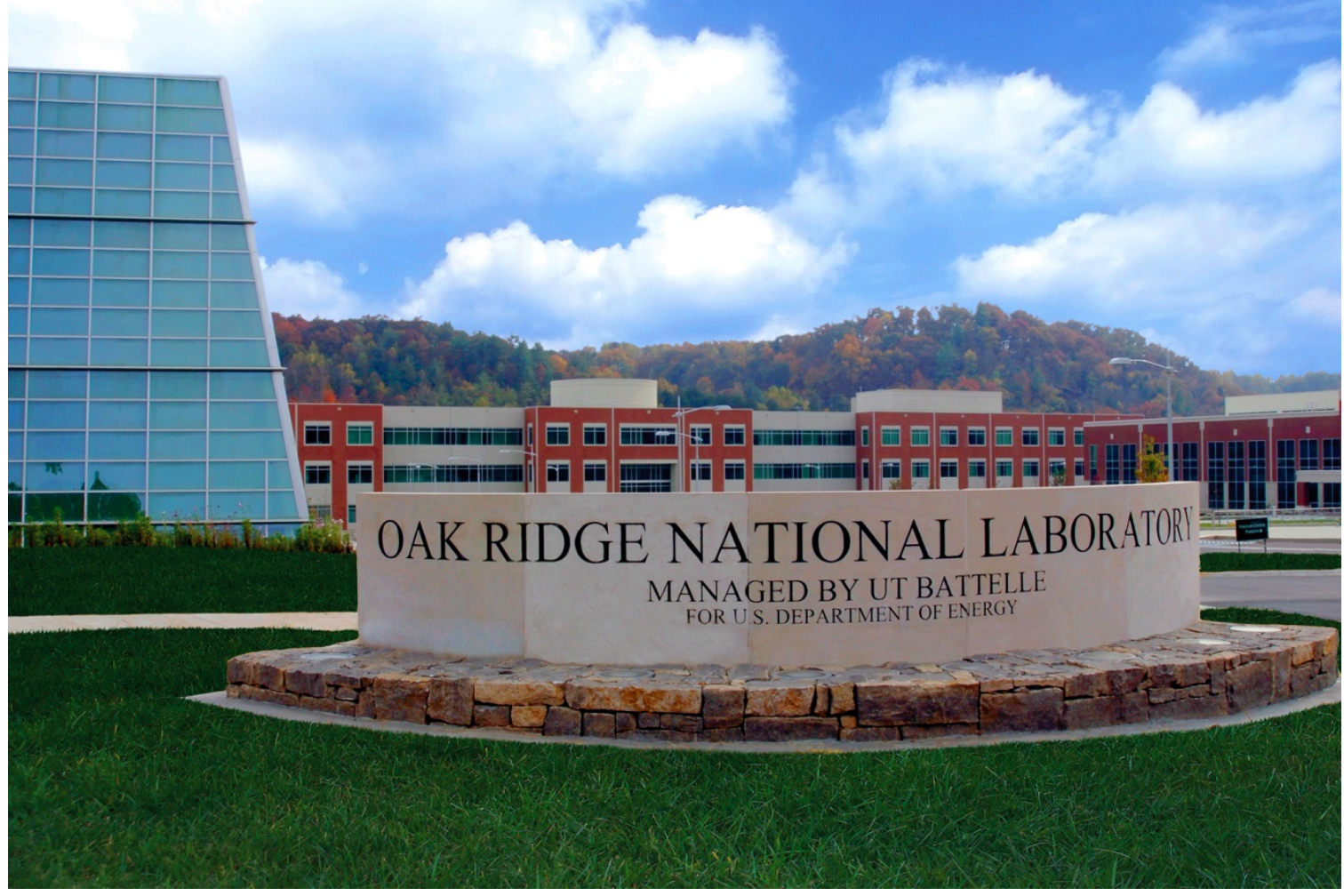
J. Rapp
T. Abrams
J. P. Allain
M. Baldwin
R. Maingi
C. Parish
M. Shimada
E. Unterberg

December 2021 


\section{DOCUMENT AVAILABILITY}

Reports produced after January 1, 1996, are generally available free via OSTI.GOV.

Website www.osti.gov

Reports produced before January 1, 1996, may be purchased by members of the public from the following source:

National Technical Information Service

5285 Port Royal Road

Springfield, VA 22161

Telephone 703-605-6000 (1-800-553-6847)

TDD 703-487-4639

Fax 703-605-6900

E-mail info@ntis.gov

Website http://classic.ntis.gov/

Reports are available to US Department of Energy (DOE) employees, DOE contractors, Energy Technology Data Exchange representatives, and International Nuclear Information System representatives from the following source:

Office of Scientific and Technical Information

PO Box 62

Oak Ridge, TN 37831

Telephone 865-576-8401

Fax 865-576-5728

E-mail reports@osti.gov

Website https://www.osti.gov/

This report was prepared as an account of work sponsored by an agency of the United States Government. Neither the United States Government nor any agency thereof, nor any of their employees, makes any warranty, express or implied, or assumes any legal liability or responsibility for the accuracy, completeness, or usefulness of any information, apparatus, product, or process disclosed, or represents that its use would not infringe privately owned rights. Reference herein to any specific commercial product, process, or service by trade name, trademark, manufacturer, or otherwise, does not necessarily constitute or imply its endorsement, recommendation, or favoring by the United States Government or any agency thereof. The views and opinions of authors expressed herein do not necessarily state or reflect those of the United States Government or any agency thereof. 
Fusion Energy Division

\title{
REPORT ON THE SECOND MPEX USER RESEARCH FORUM
}

\author{
J. Rapp \\ T. Abrams \\ J. P. Allain \\ M. Baldwin \\ R. Maingi \\ C. Parish \\ M. Shimada \\ E. Unterberg
}

December 2021

Prepared by OAK RIDGE NATIONAL LABORATORY

Oak Ridge, TN 37831

managed by

UT-BATTELLE LLC

for the

US DEPARTMENT OF ENERGY

under contract DE-AC05-00OR22725 



\section{CONTENTS}

EXECUTIVE SUMMARY

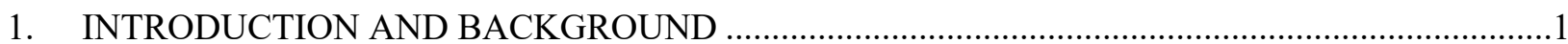

2. PMI THRUST EROSION AND REDEPOSITION ….............................................................2

2.1 GENERAL COMMENTS AND RECOMMENDATIONS FOR MPEX RESEARCH

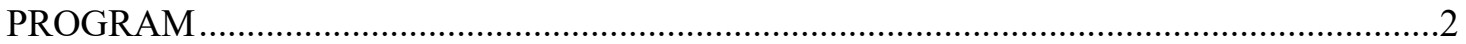

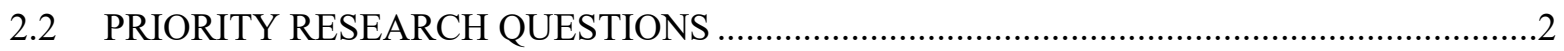

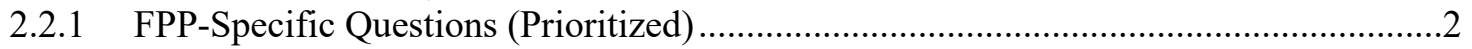

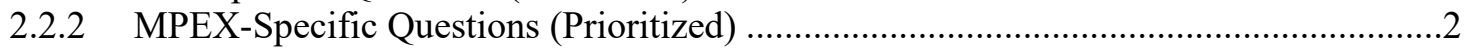

2.3 RECOMMENDATIONS AND COMMENTS ON DIAGNOSTICS NEEDS .........................

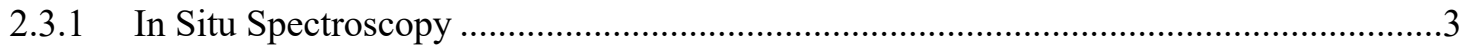

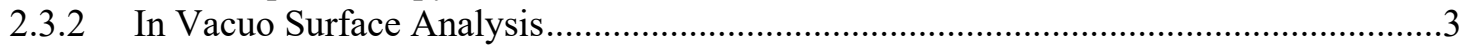

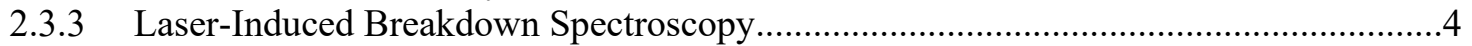

2.4 RECOMMENDATIONS AND COMMENTS ON THE TIME LINE AND

PRIORITIES OF MPEX HIGH-LEVEL COMMISSIONING ................................................

3. PMI THRUST SOLIDS AND SURFACE EVOLUTION ........................................................

3.1 GENERAL COMMENTS AND RECOMMENDATIONS FOR MPEX RESEARCH

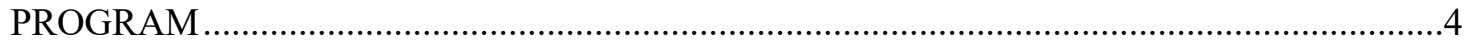

3.2 PRIORITY RESEARCH TOPICS AND QUESTIONS ..................................................

3.3 RECOMMENDATIONS AND COMMENTS ON DIAGNOSTICS NEEDS ........................6

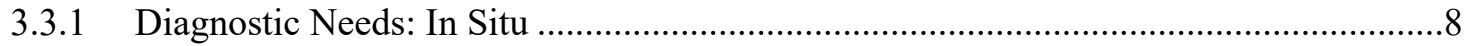

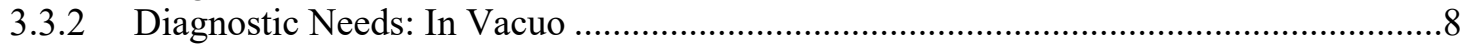

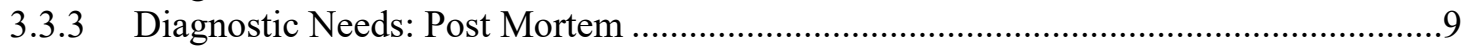

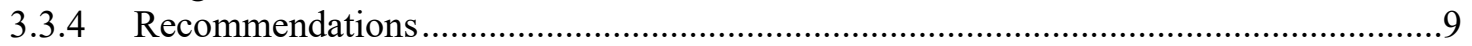

3.4 RECOMMENDATIONS AND COMMENTS ON PRIORITIES FOR MATERIAL

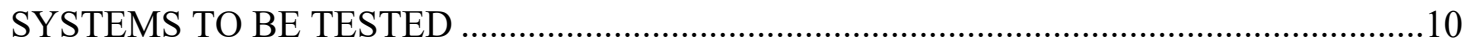

3.5 RECOMMENDATIONS AND COMMENTS ON TIME LINE AND PRIORITIES OF

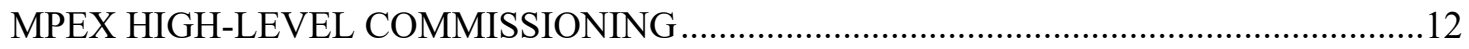

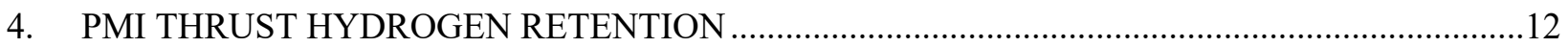

4.1 GENERAL COMMENTS AND RECOMMENDATIONS FOR MPEX RESEARCH

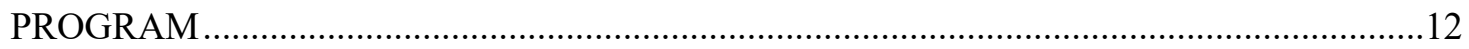

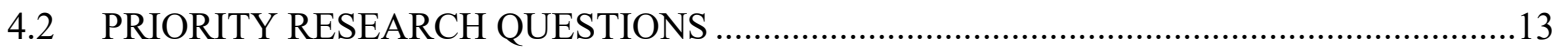

4.3 RECOMMENDATIONS AND COMMENTS ON DIAGNOSTICS NEEDS .......................14

4.4 RECOMMENDATIONS AND COMMENTS ON PRIORITIES FOR MATERIAL SYSTEMS TO BE TESTED ......................................................................................... 14

4.5 RECOMMENDATIONS AND COMMENTS ON TIME LINE AND PRIORITIES OF

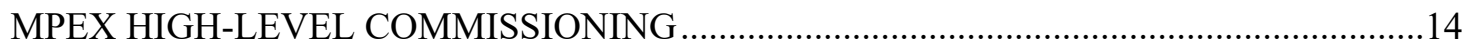

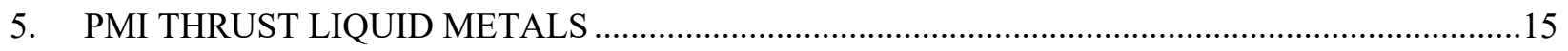

5.1 GENERAL COMMENTS AND RECOMMENDATIONS FOR MPEX RESEARCH

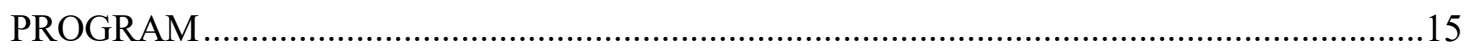

5.2 PRIORITY RESEARCH QUESTIONS …............................................................................ 15

5.3 RECOMMENDATIONS AND COMMENTS ON DIAGNOSTICS NEEDS ........................17

5.4 RECOMMENDATIONS AND COMMENTS ON PRIORITIES OF MATERIAL

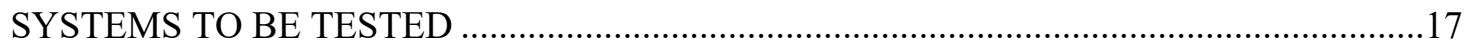

5.5 RECOMMENDATIONS AND COMMENTS ON THE TIME LINE AND

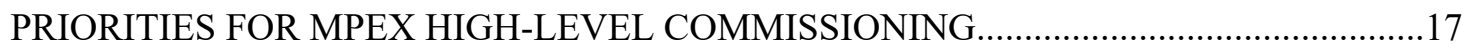

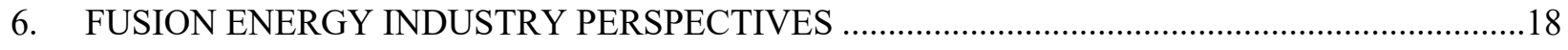

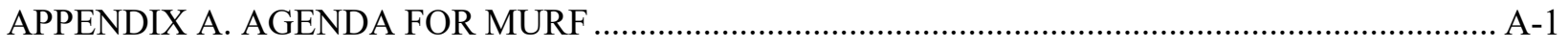




\section{EXECUTIVE SUMMARY}

The Material Plasma Exposure eXperiment (MPEX) User Research Forum (MURF), a 2 day workshop, was held virtually on September 13-14, 2021, to seek community input for MPEX, a new high-power linear plasma device that is currently being built at Oak Ridge National Laboratory (ORNL). This was the second MURF workshop. The first MURF workshop was held in October 2019 in La Jolla, California, and focused exclusively on the definition of the surface analysis station for MPEX. MPEX is designed to address crucial R\&D gaps in plasma material interactions (PMI) for future fusion reactors. The MPEX plasma source and heating systems will allow plasma exposures of plasma-facing materials and components to prototype fusion reactor divertor plasma conditions. The MPEX design accommodates the introduction of previously neutron-irradiated materials for fusion reactor-relevant plasma exposures. This capability will be unique worldwide. The US fusion program has advocated for such a device in numerous community reports in recent years, and now ORNL is building this new device. MPEX is a Major Item of Equipment project executed by the US Department of Energy's Office of Science Fusion Energy Sciences.

The purpose of this workshop was to obtain community input into the definition of the MPEX research program for its first 5 years. The workshop comprised formulation and prioritization research questions that are appropriate for a linear plasma device and can use MPEX's unique capabilities. The community was asked to provide information on how to best support the successful and timely resolution of these research questions, including topics such as:

- early exploitation in parallel to high-level commissioning, which could lead to a possible reprioritization of the commissioning program, if necessary;

- the identification of improvements for the next-generation target holder assembly;

- the identification/prioritization of diagnostics needs beyond device commissioning;

- the identification of potential material targets to be tested and reference materials throughout the phases of high-level commissioning; and

- the identification of the first batch of irradiated materials to be tested.

Furthermore, the workshop identified collaborations with confinement facilities that would leverage MPEX operations or generate unique synergies in identifying, testing, or validating candidate materials for future fusion systems.

The community identified many high-priority research questions in line with previous community workshops on PMIs. Although the research directions on the PMI of solid materials were clearly defined and the unique capabilities of MPEX acknowledged, the research directions on PMIs of liquid metals (LMs) rely on permissible in-vessel inventory of the liquid alkaline metals.

The following recommendations were made.

1. The community recommended that the MPEX team better define its operational boundary conditions for the use of alkaline metals in MPEX (e.g. in-vessel inventory, ex-vessel inventory, operating temperatures).

2. The community recommended that the MPEX team consider installing the following diagnostics in future upgrades, not prioritized:

- optical emission spectroscopy in near-UV and UV,

- hyper-spectral imaging, 
- in situ laser-induced breakdown spectroscopy,

- atomic force microscopy,

- x-ray photoelectron spectroscopy,

- ellipsometry, and

- transient gradient spectroscopy.

3. Additionally, the community stressed the need to study transient events. The community recommended that the MPEX team consider installing a laser to enable the study of transient edge-localized mode-like events.

4. The time line and priorities of MPEX's high-level commissioning were discussed. Most community members consider the correct approach to be increasing the power first and then extending the pulse length to a 2 week operation. Fusion industry representatives were concerned about the time line and advocated for a more aggressive time line. Fusion industry's emphasis is more on the long-pulse exploitation. The community recommended that the MPEX team assess whether increased funding could accelerate the high-level commissioning period. 


\section{INTRODUCTION AND BACKGROUND}

As fusion enters a phase aimed at harnessing fusion energy, new plasma-facing materials (PFMs) and components that can withstand the harsh conditions of fusion reactors must be developed. Solutions are needed for PFMs to be irradiation resistant and erosion resistant over long operation durations. The capabilities to test PFMs under fusion prototypic conditions do not currently exist. The linear plasma device called the Material Plasma Exposure eXperiment (MPEX) will close this gap by exposing a priori neutron-irradiated materials to fusion reactor prototypic divertor plasma conditions. This is achieved with a novel plasma source and heating concept, which uses a high-power helicon $(200 \mathrm{~kW}, 13.56 \mathrm{MHz})$ to create a high-density plasma that will be heated by waves in the electron cyclotron resonance frequency range $(400 \mathrm{~kW}, 70 / 105 \mathrm{GHz})$ and in the ion cyclotron resonance frequency range $(400 \mathrm{~kW}, 6-9 \mathrm{MHz})$. This concept should allow electron and ion temperatures of up to $15 \mathrm{eV}$, electron densities of up to $10^{21} \mathrm{~m}^{-3}$, and parallel heat fluxes of up to $40 \mathrm{MW} / \mathrm{m}^{2}$ to be reached in front of the target. The device will operate in steady state and use superconducting magnets to reach the required magnetic fields of $1 \mathrm{~T}$ at the target. A surface-analysis station, which allows in vacuo analysis with a large set of diagnostic tools, is an integral part of MPEX. A high-level commissioning plan to bring MPEX to its full performance, including the capability to expose activated materials and the ability to run high-fluence discharges of up to 1 million seconds, was developed. Part of this high-level commissioning plan is also a plan for a transition to R\&D operations with some of the first PMI research combined with device commissioning. This research program should focus on urgent R\&D tasks that use MPEX's unique capabilities and the plasma performance at the respective stage of the high-level commissioning.

Broadly speaking, the science and technology topics include the following.

1. Erosion, redeposition, and codeposition: Energetic ions and charge-exchange neutral fluxes cause material surface erosion. Erosion can cause out-of-design limit changes to plasma-facing components (PFCs) and affect component lifetime, and eroded products in a fusion plasma typically degrade core performance. Eroded material that is redeposited incorporates D, T, and $\mathrm{He}$ that "codeposit," thus driving T inventory and unintended changes in surface morphology. Codeposited layers can be a source of dust and flakes, which can also degrade core plasma performance.

2. Out-of-equilibrium gas implantation and surface morphology evolution: The exposure of metallic surfaces to $\mathrm{D}, \mathrm{He}$, or $\mathrm{D} / \mathrm{He}$ plasma ions leads to an out-of-equilibrium concentration of gaseous species in the PMI surface. D can cause swelling of the PMI surface in a process called blistering in which molecular recombination in large voids or grain boundaries leads to pressure buildup. He causes a temperature-dependent nanometer-scale He bubble field in the near surface that produces PMI effects, such as reduced D migration or a dense foam-like structure at low temperatures and tendril-like "fuzz" growth at higher temperatures.

3. Very low allowable T retention probability: For a DT fusion energy source to be viable, it must breed enough $\mathrm{T}$ fuel for a self-sustaining operation and excess to start up new plants. A $\mathrm{T}$ breeding ratio $>1$ defines this criterion. Materials must possess a $\mathrm{T}$ retention probability of lower than $\sim 1 \mathrm{ppm}$ in PFCs - in a reasonable amount of operational time - to meet this constraint.

4. Thermal transients: Edge-localized modes and other thermal transients are short (approximately milliseconds) unintended releases of core energy into the PFCs. The high thermal and particle loads can cause melting, ablation, crack formation, and the release of dust and flakes. 
5. Damage and transmutation effects: Defects and transmutant production caused by fusion neutrons - which, in the absence of a fusion prototypic neutron source, can be simulated by fission neutrons and/or high-energy heavy-ion bombardment — can lead to significant increases in $\mathrm{T}$ fuel retention. This can hinder the achievement of a $\mathrm{T}$ breeding ratio $>1$, causing material swelling and degrading thermal conductivity and other thermomechanical material properties, such as ductility and hardness.

The following sections detail the research topics and identify research questions for PMI for future fusion reactors. The community was asked to rank the research questions and prioritize them for the early exploitation of MPEX.

\section{PMI THRUST EROSION AND REDEPOSITION}

\subsection{GENERAL COMMENTS AND RECOMMENDATIONS FOR MPEX RESEARCH PROGRAM}

No current device can exactly replicate the full combination of conditions expected near the divertor and walls of a fusion reactor. Therefore, developing predictive models for PMIs expected in a fusion reactor validated on current experiments, such as MPEX, remains the highest priority for the program. Gaps in validated physics understanding (i.e., in which quantitative predictions do not match experimental measurements within the error bars) exist across many elements of the erosion/redeposition "chain," including the incident particle distribution functions, erosion probabilities, atomic physics rate coefficients, and prompt/nonprompt redeposition probability distributions. Because of the combination of high-target densities, grazing incidence angles, diagnostic access, and long exposure time, MPEX is in a better position than most - if not all — toroidal devices to perform careful validation studies of erosion/redeposition PMI models. The prioritized research questions that MPEX should address are listed in Section 3.2.

\subsection{PRIORITY RESEARCH QUESTIONS}

\subsubsection{Fusion Pilot Plant-Specific Research Questions}

The prioritized FPP-specific research questions that MPEX should address are as follows.

1. What is the magnitude of tritium codeposition on redeposited material and release mechanisms?

2. Eventually, in long pulse (i.e., up to 1 million s), do any "phase changes" occur to redeposited materials at very high fluence levels (e.g., dust, arcing, macroscopic delamination)?

\subsubsection{MPEX-Specific Questions}

The prioritized MPEX-specific research questions that MPEX should address are as follows.

1. What is potential profile in magnetic sheath, and how does this affect ion impact energy and angle?

- Incident ions charge state distributions are needed.

- Surface roughness/morphology dependence plus characterization.

2. What is the most reliable method for diagnosing net-erosion in situ in real time?

- MPEX can and will span parameter ranges of future reactors with relevant sheath. 
- Confirm and validate B-field effects on W prompt redeposition.

3. How accurate are atomic rate coefficients for fusion-relevant materials, particularly high-Z?

- Supplement and/or enhance Atomic Data and Analysis Structure (ADAS) calculations used for nuclear fusion applications.

- Determine the effect of sheath on $\mathrm{S} / \mathrm{XB}$ coefficients.

4. For mixed materials, how does gross erosion change at high fluence due to preferential sputtering effects?

5. What is the effect of ion implantation and neutron damage on gross erosion yields at high fluence?

6. What are the driving physics mechanisms for current and planned wall conditioning methods?

- Determine the needed surface growth and erosion of surface contamination.

- Mass flow-through of active wall conditioning methods (e.g., B powder dropping).

\subsection{RECOMMENDATIONS AND COMMENTS ON DIAGNOSTICS NEEDS}

Based on the presentations given during the workshop and the discussion that followed, the following diagnostic needs were discussed. They are presented in this section in prioritized order.

\subsubsection{In Situ Spectroscopy}

Extensive spectroscopic capability is recommended for inclusion within the MPEX diagnostic suite. The capability should include wavelengths from the visible/near-infrared and near-UV/vacuum UV, as well as have sufficient spatial resolution to resolve not only local effects of material erosion and deposition but also have resolution for measuring phenomena within the magnetic pre-sheath. Capabilities such as hyper-spectral imaging were presented and provide intriguing capabilities in this area.

Particular attention should be given to emission lines related to high- $Z$ materials, such as $\mathrm{W}$, and how MPEX could be a valuable community asset in benchmarking the atomic physics needs of these high- $Z$ elements.

Additionally, incorporating the ability to simultaneously measure many-charge states of incident and sputtered elements is a highly desired and unique capability.

\subsubsection{In Vacuo Surface Analysis}

To evaluate the effects of surface chemistry and the composition of redeposited layers, adequate surface analysis is required. Ideally, a Material Analysis and Particle Probe (MAPP)-like device would provide the expected capability in this area.

Furthermore, in situ analyses of sample surface morphology would provide additional capabilities. Diagnostics such as digital holography were presented and potentially provide the capability for in situ surface morphology and long-term net erosion measurements. 


\subsubsection{Laser-Induced Breakdown Spectroscopy}

In addition to using laser-induced breakdown spectroscopy (LIBS) for other purposes (e.g., H retention studies), these instruments are useful in erosion and migration studies for in situ depth marker analysis. Double-pulse laser options were also discussed for use in material migration (e.g., impurity transport) studies. Novel ideas based on the use of isotopic and/or elemental beam-implanted depth markers were also discussed.

\subsection{RECOMMENDATIONS AND COMMENTS ON THE TIME LINE AND PRIORITIES OF MPEX HIGH-LEVEL COMMISSIONING}

Having high power (so more diverter like target conditions) sooner as early in the high-level commissioning phase as possible would be more impactful than long-pulses. E.g., Magnum-PSI already has done very long exposures, but at low ion temperatures. This parameter regime drives the impurity sourcing better, i.e., although impurity control is going to be a challenge in long pulse, high power operations will allow to characterize all potential sources of the impurities and work on mitigation methods before absolutely needing them for long-pulse.

Another crucial capability that should be obtained as soon as possible in the commissioning phase is tilted targets. Obtaining relevant pre-sheath conditions as soon as possible in a linear device is not only relevant for transferring understanding to toroidal devices but is also a world-leading capability of MPEX.

\section{PMI THRUST SOLIDS AND SURFACE EVOLUTION}

\subsection{GENERAL COMMENTS AND RECOMMENDATIONS FOR MPEX RESEARCH PROGRAM}

For the study of PMI with solids and surfaces, MPEX will have unique capabilities compared with present linear devices and toroidal systems. The recommendations in this section are based on unique and high-priority science that will be enabled by MPEX. The unique capabilities include the following:

- the ability to expose radioactive materials,

- the ability to achieve ion impact at reactor-typical low angles of incidence $\left(\sim 5^{\circ}\right)$,

- the ability to heat ions and expose materials targets without electrical bias,

- the ability to control $T_{e}$ and $T_{i}$ independently,

- $\quad$ steady-state operation up to $10^{6} \mathrm{~s}$,

- a dedicated in vacuo surface analysis station (SAS) to examine the PMI surface without exposure to air at various times during the exposure, and

- gas fueling close to the target to study detached plasma-like conditions.

\subsection{PRIORITY RESEARCH TOPICS AND QUESTIONS}

This section summarizes the open science questions and technology issues that the users forum decided were priority research topics that could be investigated in MPEX's early operation phase parallel to the high-level commissioning. As noted by the forum, this section is expected to show considerable overlap with other sections, particularly the sections on thrusts associated with erosion, redeposition, and $\mathrm{H}$ retention. The overlap is a sign of the coupled multiphysics nature of the problems and synergies that fusion-relevant PMI presents. The research topics are listed in this section and are not necessarily in order of priority. 
The MPEX team was asked to clearly differentiate problems that will be addressed by a long-duration, low-flux plasma vs. a shorter-duration, higher-flux plasma. This is noted in the following points, when relevant.

- At least for $\mathrm{W}$, experiments at high surface temperature show that an ion-energy threshold $(\sim 10 \mathrm{eV})$ must be exceeded for a nanostructure to develop. Under detached divertor-like conditions - high density, low ion temperature, and MPEX accessibility - it is presently unclear whether fuzz will develop. A clear answer to this question will guide and inform divertor PMI predictions for any future Fusion Pilot Plant (FPP) with respect to surface evolution, erosion, redeposition, and retention.

- A natural follow-on concern is that the nature of fuzz growth in erosion- and/or redepositiondominated regimes will be caused by energetic $\mathrm{D}, \mathrm{He}$, impurity species, or a source of $\mathrm{W}$ atoms that may cause conditions that induce complicated additional fuzz growth (e.g., "super" or spiderweb-like fuzz and fuzzy trees). The more divertor-realistic inclined floating MPEX target geometry combined with low-energy heated ions gives MPEX a unique opportunity to study this effect. It would also give MPEX the potential to study fuzz melting and reintegration — or modes of degradation — caused by the effects of thermal transients if a suitable long-pulse laser system were incorporated into experiments. This will require higher-flux experiments.

- For metals operated in lower-temperature windows in which fuzz formation is not prevalent, experiments show - at least for W- that He implantation causes the uppermost tens of surface nanometers to become a dense foam-like region of He-induced nanobubbles. The nanobubbles are an effective surface barrier to deuterium diffusion, consequently reducing bulk D retention from PMI. For the same reasons as in the first topic, it is unclear whether this potentially beneficial inventoryreducing effect will be preserved in the detached divertor-like conditions possible in MPEX if $\mathrm{He}$ bubble formation in the near surface is inhibited. This will require a high-flux experiment.

- Exploratory materials with down-selection via pre-MPEX experiments should also have priority in MPEX PMI experiments, complementing the strong community focus on $\mathrm{W}$ and $\mathrm{W}$ alloys. $\mathrm{W}$ alloys should also be fabricated to examine the materials' physics of elemental transmutation caused by fusion neutron irradiation to begin understanding how realistic W-Re-Os chemistry will complicate W-H-He interplay within the material. Furthermore, such PMI experiments are expected to provide a unique ability to experimentally probe complex nuclear damage-related structures (e.g., dislocation loops, point defects, cavities, solid-solution transmutation products, intermetallic precipitates, soluterich cavity skins) that will, in turn, provide information relevant to an FPP and that are currently not readily tractable for simulation. Other fusion materials candidates (e.g., composites, ultrahigh temperature ceramics [UHTCs], $\mathrm{SiC}, \mathrm{W}$ foams) should be considered and explored in MPEX PMI experiments. This will benefit primarily from high-flux experiments.

- There is an opportunity for MPEX to make a valuable contribution to understanding PMI synergies for light ions (e.g., D, He) at extreme particle fluxes. High-flux experiments (e.g., PISCES, MAGNUM) point to the following examples.

- Sputter yields below Stopping and Range of Ions in Matter (SRIM) predictions for light ions suggest an out-of-equilibrium surface concentration effect.

- Reduced observation of $\mathrm{H}$ implantation is inconsistent with diffusion reaction code modeling of the plasma uptake using SRIM reflection values. The need to increase reflection in lockstep with flux in calculations suggests a surface $\mathrm{H}$ concentration-driven effect.

- Reduced D retention for comparable fluence is consistent with a surface concentration-driven effect of a complex interplay between flux, fluence, and exposure time.

The exceptional MPEX flux/fluence ranges combined with in situ or in vacuo surface diagnoses of $\mathrm{H}$ concentration may yield further insight and understanding of time, flux, and fluence synergies. This will require complementary high- and low-flux experiments. 
- Codeposited layers are a driver of in-vessel $\mathrm{T}$ inventory in fusion devices, as well as a potential source of dust and flakes that can lead to core contamination issues. High-flux PMI regimes in MPEX provide an opportunity to study $\mathrm{H}$ retention in down-selected priority fusion reactor PFMs. Presently, the database for codeposit properties is inadequate and limited to magnetron sputtered layers of $\mathrm{W}$ at substrate temperatures below $\sim 800 \mathrm{~K}$. The forum notes few, if any, multi-effect (e.g., damage, He, transients) studies. Studies to explore the friability and dust-generating potential of redeposition and codeposition are also deemed important. This will emphasize high-flux experiments.

- Erosion experiments in linear devices use target bias to facilitate normal incident mono-energetic ion impact. Angular incidence and distributed impact energy are difficult to study, and observations in tokamaks are often different than those found in linear devices. The ion-heating capability of MPEX, which enables a range from normal to shallow incidence ion impacts on materials targets, provides an excellent opportunity to bridge this gap. Other PMI effects, such as morphology development (e.g., cones, fuzz), can be studied simultaneously.

- PFC performance crucially relies on a material's thermomechanical properties for effective heat removal. Lattice imperfections (e.g., vacancies, defects, grain boundaries) scatter electrons and phonons in materials and consequentially reduce thermal conductivity and diffusivity. Experiments and calculations show that dissolved $\mathrm{D}$ and He from plasma exposure or nuclear processes can stabilize material defects that might otherwise anneal, and these unannealed defects contribute excess thermal conductivity loss. The forum noted that MPEX experiments provide a unique platform to begin studying the physics of this issue and also provide a test bed for material model validation of neutron damage. Examining synergisms was noted as important because differences between sequential vs. simultaneous experiments often occur (i.e., target material damaged before plasma exposure vs. damage during plasma exposure), as were differences in target evaluation if performed in situ or ex situ vs. in operando. The newly developed techniques of in operando LIBS and transient gradient spectroscopy (TGS) could prove useful in addressing such differences. This will be most directly addressed with low-flux experiments.

- Thermal transients in fusion devices are rapid (approximately millisecond time scale) dumps of core energy into the PFCs. The forum discussed options for such experiments on MPEX, noting that PMI experiments - including this effect — might be considered with appropriate upgrades. Although not currently planned, adding an appropriate long pulse source of transient thermal loading to MPEX targets (e.g., a laser) might be used to study the effects of thermal shock on ablation, erosion, toughness, cracking, and other types of fracture or degradation modes. Effects on resultant materials' surface composition are highly relevant, as is testing emerging materials with enhanced shock resistance, such as composites (e.g., UHTCs), additively manufactured materials, and solid foams (e.g., W foam). This would involve low- and high-flux experiments.

\subsection{RECOMMENDATIONS AND COMMENTS ON DIAGNOSTICS NEEDS}

The priority research questions in this section frame categories of material effects that require diagnosis either during or subsequent to (i.e., postmortem) the plasma exposure. Although a thorough discussion on these needs was outlined in the first MURF, a brief synopsis is given here because these techniques were discussed in presentations and discussions at the second MURF. 
Diagnosis techniques, methods, and property types for MPEX fusion-relevant PMI materials are broadly classified as follows:

- $\quad$ surface morphology (e.g., roughness, nanoscopic fuzz tendrils/fuzz layer thickness, pinhole size and density),

- $\quad$ surface chemistry (e.g., contamination layers, local H/He content, migration of isotopically tagged marker layers, chemical binding of elements),

- $\quad$ subsurface morphology (e.g., bubble sizes, bubble densities, blister morphology),

- $\quad$ subsurface chemistry (e.g., mixing, H/He depth profiles, transmutation composition),

- mechanical properties (e.g., layer adhesion, hardness, brittleness, ductility, elastic modulus), and

- thermal properties (e.g., thermal conductivity, thermal diffusivity).

MPEX diagnostics for the characterization of these effects can be separated into three broad classes.

1. Performed in the MPEX plasma chamber with either the plasma on (i.e., in operando) or off (i.e., in situ). These techniques are the most difficult because they must be integrated into the MPEX system, but they will be most representative of the "in-service" state of the material.

2. Performed without breaking vacuum or must be performed without disturbing the sample/holder coupling (i.e., in vacuo) but not in the plasma chamber. Such techniques will be performed in the MPEX SAS. The SAS vacuum and transfer will allow near-pristine surfaces to be analyzed without air exposure and highly reduced adventitious layer formation. This will also not require the sample to be removed from the thermal transfer assembly so that interrupted experiments can be performed, returning the sample to the plasma chamber for further exposure. Removing the sample from the thermal transfer assembly is undesirable because the surface temperature could change during subsequent exposures.

3. Performed after plasma exposure and with exposure to air (i.e., postmortem) in a separate laboratory. Postmortem analyses involve transporting the sample from the MPEX facility to another facility and will enable the largest variety of techniques but do not permit the pristine surface to be preserved. Thus, further MPEX exposures of the sample could result in changes to the thermal behavior of the sample by removing and replacing it in the sample holder.

Table 1 summarizes a range of diagnostic capabilities that the forum recommends as crucial for measuring the effects on MPEX PMI. 
Table 2. Forum-recommended MPEX diagnostic methods. .

\begin{tabular}{|c|c|c|c|}
\hline & $\begin{array}{c}\text { In situ } \\
\text { (MPEX PMI } \\
\text { chamber) }\end{array}$ & $\begin{array}{c}\text { In vacuo } \\
\text { (MPEX SAS) }\end{array}$ & $\begin{array}{c}\text { Postmortem } \\
\text { (external laboratories) }\end{array}$ \\
\hline Surface morphology & Ellipsometry & $\begin{array}{l}\text { Scanning electron microscopy } \\
\text { (SEM), atomic force microscopy } \\
\text { (AFM), ellipsometry }\end{array}$ & SEM, AFM, ellipsometry \\
\hline Surface chemistry & & $\begin{array}{c}\text { SEM energy dispersive x-ray } \\
\text { spectroscopy (EDX), x-ray } \\
\text { photoelectron spectroscopy (XPS) }\end{array}$ & $\begin{array}{c}\text { SEM-EDX, secondary ion } \\
\text { mass spectroscopy } \\
\text { (SIMS), XPS } \\
\end{array}$ \\
\hline Subsurface morphology & & SEM focused ion beam (FIB) & $\begin{array}{c}\text { FIB, transmission electron } \\
\text { microscopy }\end{array}$ \\
\hline Subsurface chemistry & $\begin{array}{c}\text { LIBS/laser } \\
\text { ablation mass } \\
\text { spectroscopy } \\
\text { (LAMS), ion } \\
\text { beam analysis } \\
\text { (IBA) }\end{array}$ & LIBS/LAMS, IBA & $\begin{array}{l}\text { SIMS, LIBS/LAMS, } \\
\text { thermal desorption } \\
\text { spectroscopy (TDS), IBA }\end{array}$ \\
\hline Mechanical properties & & AFM & AFM, nanoindentation \\
\hline Thermal properties & TGS & & TGS \\
\hline
\end{tabular}

\subsubsection{Diagnostic Needs: In Situ}

The forum noted several opportunities for MPEX PMI studies that could take advantage of in operando diagnoses. Studies of near-surface thermal conductivity and out-of-equilibrium effects caused by the saturation of the surface with D and He would require TGS and LIBS, respectively. TGS uses a pump/probe laser and detection system that measures a nondestructively induced thermal transient surface acoustic wave, as well as gives a measure of thermal diffusivity and elastic modulus. However, in situ TGS is still only in its infancy and will likely be less effective for fluences that may see morphology development (e.g., fuzz growth or roughening/pinhole formation). Ellipsometry is a potential tool for measuring surface roughness in situ and possibly in operando, and it will be valuable for validating models of surface layer growth and roughening. One way to measure $\mathrm{D} / \mathrm{He}$ retention under the surface could take advantage of an in situ quantified LIBS setup or in situ - potentially in operando-IBA and would also greatly help validate surface composition for modeling efforts. However, integrating ellipsometry or IBA onto MPEX's plasma chamber will likely require a longer-term, supplementally funded upgrade path.

\subsubsection{Diagnostic Needs: In Vacuo}

In vacuo techniques are expected to provide much of the early MPEX science. SEM is tentatively planned for the MPEX SAS. Using SEM at interrupted fluence levels will allow the morphology of the evolving structure to be captured. For instance, fiducial marks could be introduced onto the sample before exposure using FIB or laser scribing, and these marks are found at progressively increasing plasma fluence to capture the evolving states of the specimen. Adding EDX capabilities to the SEM is relatively simple. EDX collects the $\mathrm{X}$-rays generated by the fall of the SEM beam on the specimen and can identify the elements of $Z=5$ (B) and heavier. SEM-EDX is nondestructive and will easily identify gross chemical changes (down to about $1 \mathrm{wt} \%$ ) in the surface (a few hundred nanometers). XPS can be used to provide much better detection limits $(<1 \mathrm{wt} \%)$ and also provides bonding information, but the vacuum levels achievable in the MPEX SAS might be insufficient $\left(<10^{-9}\right.$ Torr $)$ to keep a surface clean without 
monolayer formation from residual gases. AFM uses a small physical probe to measure the height of a surface with high precision (potentially $<1 \mathrm{~nm}$ if $z$-resolution and a few nanometers if $x y$-resolution), and it may be possible to be incorporated into the SAS. AFM will complement SEM by providing quantitative surface roughness but will have less versatility than SEM.

Examining the subsurface (i.e., greater than a few nanometers) will require destructive techniques. FIB can mill small (approximately tens of microns) craters that, with proper attention to chamber geometry, will allow SEM to examine gross features (e.g., fuzz, blisters) up to a few dozen microns beneath the surface. LIBS/LAMS methods use a focused laser to ablate a crater into the surface and analyze the resultant plumes and can, in principle, measure the D or He under the surface, although depth resolution will be approximately hundreds of nanometers and have sensitivities of approximately hundreds of parts per million or worse. Investment in state-of-the-art femtosecond lasers may improve this.

IBA can provide depth profiles by using a high-energy ion beam at a glancing angle and capturing the scattered ions. IBA is ideal for depth profiling D, but the ion beam and the geometry for the analysis may be difficult to mate to the first-generation SAS. AFM and related tools, such as nanoindentation, can use nanohardness or nanoscratching methods to study the hardness, modulus, adhesion, and other mechanical properties of the surface or redeposited layers.

\subsubsection{Diagnostic Needs: Postmortem}

Postmortem methods will provide the most versatility. Dedicated AFM, SEM, and SEM-FIB systems will provide performance superior to SAS-integrated systems and will enable a detailed analysis of the postexposure state of the samples to be performed. SEM-FIB can be used to prepare samples for TEM that will enable a detailed examination of the surface layers, elemental analysis of layers, sizing of $\mathrm{He}$ bubbles, and other nanometer-scale structural and chemical states of the material to be performed. LIBS/LAMS postmortem can provide the depth profiles of the $\mathrm{D}$ and He with at least modest sensitivity and depth resolution. SIMS will be much more sensitive to the entire periodic table and will likely be useful for studying the redeposition and for including the transport of isotopically tagged layers from the sample to the sample hold-down assembly. Nanoindentation and nanoscratching methods could also be valuable for examining the adhesion, hardness, and friability of the redeposition layers.

\subsubsection{Recommendations}

In terms of in situ techniques within the plasma chamber, TGS should be considered as a likely upgrade path, and appropriate ports should be reserved for future investment but not for early MPEX science. Although not technically a diagnostic, a laser that delivers transient heat loads to the specimen during plasma exposure will also be valuable and should be strongly considered for early MPEX science. This will allow edge-localized mode-like heat loads to be delivered to the sample surface during plasma exposure.

Given these possible capabilities, the most important in vacuo SAS diagnostic needs recommended for early MPEX science will be, in approximately descending order, as follows:

- SEM plus EDX in the SAS chamber to measure the gross changes (morphological and elemental) on the surface,

- $\quad$ LIBS/LAMS in the SAS chamber to measure D/He under the surface, and

- $\quad$ SEM-FIB capabilities in the SAS chamber to image gross subsurface changes. 
Additional SAS needs that would be beneficial but are not necessarily easy to incorporate into a firstgeneration SAS and would be recommended for consideration in a second-generation SAS include the following:

- the capability to extract FIB lift-outs to capture the subsurface structure at high resolution via ex situ TEM,

- AFM to quantify the surface roughness,

- XPS to obtain chemical bonding and more surface-sensitive elemental information (requires a higher base vacuum level for the SAS), and

- IBA for depth profiling D to complement the LIBS/LAMS.

Postmortem needs for SEM-EDX, FIB-SEM, and TEM are available already at ORNL's Low Activation Materials Design and Analysis laboratory. SIMS, XPS, AFM, and nanoindentation/nanoscratching capabilities must be enhanced. For non-neutron-irradiated campaigns, SIMS and XPS could be obtained via collaborations within ORNL, whereas AFM and nanoindentation capabilities are modest investments for which MPEX should consider obtaining dedicated systems.

\subsection{RECOMMENDATIONS AND COMMENTS ON PRIORITIES FOR MATERIAL SYSTEMS TO BE TESTED}

The very earliest MPEX exposures might focus on well-understood materials systems, specifically wrought and single-crystal W, to provide performance baselines that can be compared with other plasma devices (e.g., PISCES, Magnum-PSI) to establish the basic operating parameters. However, new scientific ground should also be broken beyond the well-established baseline materials. The forum discussed two broad categories: (1) complex W-based materials and (2) exploratory, non-W materials.

Although one major motivation for MPEX is analyzing neutron-irradiated materials and thus beginning to unravel the plasma, irradiation, and transmutation synergies, the early MPEX campaigns will not use neutron-irradiated materials. To lay the groundwork for later neutron-irradiated campaigns, early MPEX experiments should focus on (1) engineered W-Re-Os alloys heat-treated to produce intermetallics as similar as possible to the structures observed in the neutron-irradiated samples, (2) W composites, and (3) ion-irradiated W, alloys, and composites.

W-Re-Os alloys should be designed and fabricated soon, potentially with preliminary plasma exposures in current devices for exploratory studies. Because of the copious and profligate transmutation of W under neutron irradiation, $\mathrm{W}$ is not necessarily a relevant material; transmutation must be considered for any reactor-relevant considerations. Although the precipitates formed via thermal processing will not be the same as those formed under ion irradiation, this can simulate effects such as high densities of traps at internal interfaces, like those seen in the neutron-irradiated case. Similarly, ion beam irradiationspreferably with $\mathrm{W}$ ions to minimize the generation of traps via solute atoms - will be important for early retention studies in MPEX, and choices of substrates and ion irradiation condition matrices should proceed soon.

Currently, the development of $\mathrm{W}$ composite materials and additively manufactured $\mathrm{W}$ materials is proceeding in the US fusion program. Although these materials are not yet reactor optimized, the most promising candidates should be exposed in MPEX early science programs. To prepare for this, appropriately sized samples should be neutron irradiated soon so that they have sufficient cooling (i.e., radioactive decay) time before MPEX radiological operations. 
The space of possible materials other than $\mathrm{W}$ is large, but candidates that currently appear most promising are $\mathrm{SiC}$ composites and UHTCs. $\mathrm{SiC}$ composites leverage the aerospace and fission industries in which these composites have high technology readiness levels. For instance, $\mathrm{SiC}$ composites are already used in commercial jet engines (i.e., TRL 9). SiC and composites have excellent neutron irradiation resistance, at least in fission environments. $\mathrm{SiC}$ is also promising because of its lower chemical erosion than graphite and likely lower codeposition than graphite. $\mathrm{SiC}$ composites could be considered for either divertor or main-wall applications, and this will depend on details that have not yet been determined. MPEX will be important for exploring $\mathrm{SiC}$ composites because of the limited high-flux, high-fluence data from plasma exposure of SiC-based materials. MPEX's helicon source won't be susceptible to C contamination, making high flux and high fluence more feasible. It will also be important to study combined $\mathrm{W} / \mathrm{SiC}$ targets to understand $\mathrm{SiC}$ redeposition onto $\mathrm{W}$, as would be seen in a mixed chamber.

UHTCs - particularly $\mathrm{TiB}_{2}, \mathrm{ZrB}_{2}, \mathrm{TiC}$, and $\mathrm{ZrC}$ - are leveraged from the aerospace industry, specifically reentry bodies, and preliminary neutron-irradiation results indicate promising neutron resistance. These UHTCs are particularly promising for potential fusion applications because of their very high retained strength at high temperatures and their excellent resistance to irradiation-induced amorphization. In the short term (i.e., before MPEX commissioning), preliminary studies using existing linear devices should be performed to determine whether any of the beyond-W materials that may be suggested show acceptable plasma performance; then, pristine samples and neutron-irradiated samples should be prepared for MPEX exposures. UHTC composites may become available in the near future and should also be leveraged. Currently, the main problem with UHTC concepts is that there is insufficient relevant plasma exposure data to begin understanding their behavior and limitations, so short-term pre-MPEX studies are particularly needed. Preliminary neutron-irradiation studies on carbide and diboride UHTCs have shown excellent promise.

Broadly speaking, recommendations for current activities to support MPEX science include the following.

- Choose appropriate W alloys and composites and begin High Flux Isotope Reactor irradiations as soon as possible to have MPEX samples ready with appropriate radiological cooling for the MPEX radiological campaigns.

- Perform prescreening on SiC and UHTC candidates by using current plasma devices to determine appropriate materials for future MPEX studies.

- Based on the preliminary plasma studies on SiC and UHTCs, perform High Flux Isotope Reactor irradiations to have samples cooled and ready for the MPEX radiological campaigns.

Ultimately, no single material will have the combination of properties (e.g., neutron resistance, fuel pickup, core plasma degradation, residual radioactivity) needed for FPP PFMC service, and engineered composites or complex multicomponent systems will be needed. Early MPEX science must focus on identifying the building blocks that will enable engineering designs of the needed components. 


\subsection{RECOMMENDATIONS AND COMMENTS ON TIME LINE AND PRIORITIES OF MPEX HIGH-LEVEL COMMISSIONING}

Overall, recommendations for the Solids and Surfaces thrust can be summarized as follows.

- Diagnostics of high, early-science priority include:

- SEM and EDX in the SAS for in vacuo,

- LIBS/LAMS in the SAS for in vacuo, and

- AFM/nanoindentation available for postmortem and ex situ.

- Materials for high-priority pre-MPEX and early-MPEX studies include:

- single-crystal and wrought high-purity W to establish baselines,

- W-Re-Os alloys heat-treated to simulate neutron irradiation,

- W-based composites and advanced-manufactured structures,

- $\mathrm{SiC}$ composites, and

- UHTC materials.

In vacuo SEM and EDX will be vital for the SAS to capture the evolving surface structures. If feasible, an FIB column should be included to enable milling craters to also observe the gross subsurface structures evolving. In vacuo LIBS/LAMS in the SAS will be very valuable to allow $\mathrm{D} / \mathrm{He}$ content to be examined in the sub-surface. Postmortem AFM and nanoindentation combined with existing tools (e.g., SEM) will be needed to evaluate quantitatively the structures formed by the plasma exposure.

MPEX provides an exciting new exploratory space for many materials systems. "Typical" W materials should be exposed first to establish the system baseline by comparison with other linear devices.

However, the earliest scientific yield will come from W-Re-Os alloys intended to simulate neutronirradiated structures, "advanced" W materials, and exploratory ceramic materials that may compliment metallic materials for high-heat environments.

\section{PMI THRUST HYDROGEN RETENTION}

\subsection{GENERAL COMMENTS AND RECOMMENDATIONS FOR MPEX RESEARCH PROGRAM}

$\mathrm{H}$ retention is critical in tritium in-vessel inventory assessments and PFCs material selections for licensing a fusion pilot plant. PMI determines the boundary conditions for diffusing $\mathrm{H}$ isotopes in PFCs, and research needs must elucidate the complex interplay between $\mathrm{H}$ migration/trapping and neutroninduced radiation damage/solid transmutation in reactor-relevant long-pulse plasma and neutron fluxes and fluences conditions. Historically, linear plasma devices (e.g., PISCES-A/B at University of California San Diego, Tritium Plasma Experiment at Idaho National Laboratory) had been extensively used to advance researchers' understanding of $\mathrm{H}$ behavior in PFCs with a series of well-controlled and welldiagnosed experiments, and linear plasma devices are best suited to address this PMI thrust because they can more easily reach reactor-relevant parameters, such as high ion fluxes and high ion fluence with reactor-relevant high ambient material temperatures. Additionally, linear plasma devices are more flexible than toroidal devices, allowing for controlled plasma exposure conditions and diagnostic access for independent parameter variations in the relevant PMI conditions and the testing of novel material and hazardous neutron-irradiated materials.

MPEX will provide unique plasma conditions, such as exposure to reactor-relevant ion fluxes greater than $10^{23} \mathrm{~m}^{-2} \mathrm{~s}^{-1}$. MPEX will also achieve reactor-relevant fluences on the order of $10^{31} \mathrm{~m}^{-2}$, exposure to reactor-relevant power fluxes greater than $10 \mathrm{MW} / \mathrm{m}^{2}$, the ability to operate with an unbiased and inclined target at an angle as low as $5^{\circ}$, and the ability to expose radioactive and hazardous materials, such as 
neutron-irradiated materials and LMs along with diagnostic capabilities to monitor the surface evolution and morphology changes during high-fluence exposures with a variety of surface diagnostics, including electron microscopy (i.e., in situ or in vacuo). The following recommendations were made during the $\mathrm{H}$ retention session for the MPEX research program.

- Well-controlled experiments in linear plasma devices are best suited to advance the scientific understanding of $\mathrm{H}$ retention in PFCs.

- The unique MPEX capability of high-fluence $\left(<10^{31} \mathrm{~m}^{-2}\right)$ exposure is extremely valuable for investigating $\mathrm{H}$ retention behavior during a PFC's lifetime with high-level ( $>10 \mathrm{dpa})$ neutronirradiated PFCs.

The H retention session's recommendations and feedback for MPEX's five assessments are as follows.

- Reprioritization of the commissioning program is not needed to advance $\mathrm{H}$ retention $\mathrm{R} \& \mathrm{D}$, and no urgency to accelerate $\mathrm{H}$ retention $\mathrm{R} \& \mathrm{D}$ was identified from the community or private fusion industry partners.

- Community input and discussion are needed to improve the handling of highly neutron-activated materials.

- Recommendations on $\mathrm{H}$ measurement diagnostics for $\mathrm{H}$ retention study are provided in Section 5.3.

- $\mathrm{W}$ and $\mathrm{W}$ alloys are the primary candidate materials discussed in the $\mathrm{H}$ retention session, and linear plasma devices are suitable for testing other novel materials.

- Community input and discussion are needed to determine the first batch of materials and irradiation condition.

In parallel, the community must demonstrate the validity of the sequential irradiation/exposure approach (i.e., neutron irradiation, then plasma exposure) with a simultaneous irradiation/exposure approach or modeling before performing high-level ( $>10 \mathrm{dpa}$ ) neutron irradiation.

\subsection{PRIORITY RESEARCH QUESTIONS}

Linear plasma devices (e.g., PISCES-A/B at University of California San Diego, the Tritium Plasma Experiment at Idaho National Laboratory) have been extensively used to improve researcher understanding of $\mathrm{H}$ behavior in PFCs with a series of well-controlled and well-diagnosed experiments. MPEX will further advance the PMI physics on $\mathrm{H}$ retention with the following unique capabilities:

- high-fluence operation (up to $10^{31} \mathrm{~m}^{-2}$ ),

- normal incidence and oblique incidence exposures,

- target temperature control,

- impurity in plasma (He ion) and gases ( $\mathrm{N}$ as seeding), and

- $\quad$ high $n_{e}$, low $T_{e}$, and low $T_{i}$ for a detached divertor vs. high $T_{e}$, high $T_{i}$, and low $n_{e}$ for an attached divertor operation. 


\subsection{RECOMMENDATIONS AND COMMENTS ON DIAGNOSTICS NEEDS}

PMI determines the boundary conditions for diffusing H isotopes in PFCs, and in situ and/or in vacuo diagnostic capabilities are critical for elucidating the complex surface chemistry that affects $\mathrm{H}$ behavior in PFCs for $\mathrm{H}$ retention studies. Therefore, the following diagnostics were recommended in the $\mathrm{H}$ retention session to advance $\mathrm{H}$ retention R\&D for the MPEX research program.

- In vacuo: TDS to measure total D retention.

- In vacuo: Nuclear reaction analysis to measure near-surface $(<5 \mu \mathrm{m}) \mathrm{D}$ depth profile.

- In vacuo: Auger electron spectroscopy and in vacuo XPS to examine surface chemistry and nearsurface depth profile before and after MPEX plasma exposure.

- Ex situ: Lifetime and Doppler broadening positron annihilation spectroscopy to characterize microstructural evolution in neutron-irradiated PFCs.

- In situ: LIBS and LAMS to measure near-surface H/D and impurity depth profiles.

- Ex situ: Glow-discharge optical emission spectroscopy for H/D and impurity depth profiles.

\subsection{RECOMMENDATIONS AND COMMENTS ON PRIORITIES FOR MATERIAL SYSTEMS TO BE TESTED}

Linear plasma devices are more flexible than toroidal devices, allowing novel materials and hazardous neutron-irradiated materials to be tested. $\mathrm{W}$ and $\mathrm{W}$ alloys were the primary candidate materials discussed in the $\mathrm{H}$ retention session, and linear plasma devices are suitable for testing other novel materials, such as ceramic (e.g., SiC). Community input and discussion are needed to determine the first batch of materials and the irradiation condition. In parallel, the community must demonstrate the validity of sequential irradiation and/or exposure approaches (i.e., neutron irradiation, then plasma exposure) with simultaneous irradiation and/or exposure approaches or modeling before performing high-level ( $>10 \mathrm{dpa})$ neutron irradiation.

\subsection{RECOMMENDATIONS AND COMMENTS ON TIME LINE AND PRIORITIES OF MPEX HIGH-LEVEL COMMISSIONING}

MPEX will provide research opportunities for investigating $\mathrm{H}$ retention with small coupon samples from the C1 (2027) campaign and will test large PFC components in the C7 (2029) campaign. During the C6 (2028) campaign, MPEX will investigate $\mathrm{H}$ retention in the $10^{29} \mathrm{~m}^{-2}$ fluence regime that has not been explored before with $8 \mathrm{~h}$ operation. During the C11 (2030) campaign, MPEX will test initial sets of neutron-irradiated material with $10^{30} \mathrm{~m}^{-2}$ fluence with $36 \mathrm{~h}$ exposure. Community input and discussion are needed to determine the first batch of materials and the irradiation conditions. The first batch of neutron irradiation must be completed in the 2027-2028 time frame to allow enough cooling time for radioactivity and/or the dose rate to decay to the acceptable level in the MPEX facility. During the C15 (2030) campaign, MPEX will investigate $\mathrm{H}$ retention in end-of-life $\left(10^{31} \mathrm{~m}^{-2}\right.$ fluence $)$ PFCs. Again, community input and discussion are needed to determine the materials and the irradiation conditions for end-of-life $(>>10$ dpa) PFCs.

Additionally, the following comments and questions were raised during the $\mathrm{H}$ retention session.

- The need for PFC testing should be identified beyond small material sample exposures with typical specimen dimensions of $\sim 1 \mathrm{~mm}$ thick with a 5-12 $\mathrm{mm}$ diameter, which would require target holder modifications.

- Community input and discussion are needed to determine the first batch of materials, irradiation conditions, and sizes.

- Research questions goal-oriented toward a fusion pilot plant must be identified. 
- The validity of the sequential irradiation/exposure approach must be demonstrated via a simultaneous irradiation/exposure approach, which should include modeling and experimental R\&D for $\mathrm{H}$ retention in dust and dust in PFC gaps.

- Multiphysics $\mathrm{H}$ modeling must be validated.

- The allowable tritium inventory for licensing a fusion pilot plant must be discussed and determined.

\section{PMI THRUST LIQUID METALS}

\subsection{GENERAL COMMENTS AND RECOMMENDATIONS FOR MPEX RESEARCH PROGRAM}

A session on possible LM experiments was held at the second MURF. The session included an introduction to and specific talks on flowing systems, stagnant/creeping systems, plasma response validation needs, and reactor design integration.

Although the first discussion was lively and informative, specific experiments require further assessment given MPEX's intended and planned capabilities. Furthermore, the LM user group advocated for a refined look at the LM inventory limits within MPEX and the MPEX building as a guide to the scope of possible MPEX experiments. Implications could signify a balance between the free-surface flow of liquid Li experiments with limited liquid flow and static Li experiments in MPEX.

\subsection{PRIORITY RESEARCH QUESTIONS}

LM PFC designs are at low technical readiness levels (1-3) with respect to solid PFC designs (4-6 or more) for high-power fusion devices. As such, the basic questions of LMs include a wide range of engineering, materials, and physics issues, such as:

- LM chemistry;

- wetting and drying LM on the substrates;

- corrosion/embrittlement of the LM with the substrate;

- neutron damage to solid-based substrate materials (common with solid PFCs);

- transport across magnetic fields of conductive, flowing LM concepts;

- tritium transport, retention, recovery, and multiphase flow in flowing LM systems;

- He exhaust handling in LM systems;

- H accounting recycling, pumping/recovery (possibly multiphase flow and fueling);

- identifying heat flux limits and operational temperature limits of flowing LMs, including capillary porous systems (CPS);

- surface vapor shielding, Li recirculation, continuous volumetric detachment, and their integration;

- compatibility of LMs with high-performance plasma confinement validation of models with MPEX experiments;

- characteristics of the LM vapor cloud for high surface temperature designs; and

- sheath effects on impingement and release of LM from surfaces.

Most of these would be best addressed in dedicated test stands. One important consideration is that because water is used in the MPEX facility, a limit of $3.45 \mathrm{~mol}$ of LM was established for MPEX (which may be doubled to be $6.9 \mathrm{~mol}$ as the lower explosive limit is based on hydrogen molecules and not atoms as used for the $3.45 \mathrm{~mol}$ limit), which is $\sim 25-50 \mathrm{~g}$ of liquid Li. The primary task is to identify LM experiments that exploit the unique capabilities and diagnostics of MPEX within the prescribed site alkali metal inventory limits. 
The R\&D needs for flowing systems were discussed. These needs include investigations of recycling and pumping because Li pumps hydrogenic species, the recovery of $\mathrm{H} / \mathrm{D}$ ( $\mathrm{T}$ surrogates) from the liquid Li stream, He pumping schemes, and power exhaust capabilities. The following flowing LM concepts were discussed:

- FLiLi, which uses gravity and jxB pumps to circulate the flow;

- LiMIT, which uses TEMHD to augment/supplant gravity drive;

- CPS, which has fast flow in channels near the substrate; and

- divertolets, which create a short flow path to reduce required flow speed.

Additionally, two methods for hydrogenic species removal from flowing Li were mentioned: a LiH separation magnetic centrifuge and a distillation column. The general needs from the MPEX facility were outlined as (1) Li handling and flow systems that feed into MPEX, (2) stationary and mobile glove boxes, and (3) Li loops and related $\mathrm{Li}$ injection systems.

During the meeting, R. Goldston discussed specific ideas for stagnant or creeping liquid Li systems. Three specific experiment concepts were proposed, as well as a fourth that does the first three simultaneously.

1. Continuous surface vapor shielding: Operate for long pulses and demonstrate continuous external feed of Li during the pulse from a feed ring around the target. MPEX requires (1) a Li reservoir in contact with feed ring around target, (2) $7.5 \mathrm{~g}$ of Li evaporated into vessel, and (3) a pulse length of minutes to $1 \mathrm{~h}$ based on Magnum-PSI experience.

2. Real-time Li recirculation: Surround the target area with warm CPS and demonstrate Li condensation. MPEX requires a condenser cylinder at $\sim 300^{\circ} \mathrm{C}$ that is $\sim 10 \times 7 \times 2 \mathrm{~mm}$ and comprises $11 \mathrm{~g} \mathrm{Li}$.

3. Continuous volumetric detachment: Form a vapor box around the MPEX beam and operate continuously with volumetric detachment. MPEX requires a temperature of $650^{\circ} \mathrm{C}$ and a condenser.

4. Simultaneous achievement of the aforementioned experiments: MPEX requires a longer system with an evaporator and condenser.

The needs to address open questions in plasma modeling of Li PFCs for the Fusion Nuclear Science Facility (FNSF) were discussed. The boundary plasma of an FNSF design is being simulated as part of the domestic LM PFC concept development program. The SOLPS-ITER code is used for simulations, including $\mathrm{Ne}$ gas, to reduce the upstream heat flux and $\mathrm{Li}$ evolution from targets. Cross-field transport rates are chosen to yield a $5 \mathrm{~mm}$ scrape-off layer heat flux width. The Li evaporation rate from the surface is based on a sheath model calculation provided by D. Curreli's group at the University of Illinois at Urbana-Champaign. The first set of simulations shows very efficient Li entrainment in the divertor (i.e., with $<1 \% \mathrm{Li}$ concentration outside the divertor). This would be very favorable for avoiding $\mathrm{Li}$ contamination in the main chamber plasma. The simulation has open questions that could be addressed by MPEX, such as the source of intrinsic impurities from the surface, parallel impurity ion transport physics, and Li pumping of $\mathrm{H}$. Specific experimental needs include:

- Li source as function of surface temperature, main ion flux, impurity fluxes, neutron damage, and target angle;

- impurity retention under strong main ion flow (i.e., reactor-level) conditions; and

- H retention of flowing Li under reactor conditions. 
Finally, integration aspects with reactor designs were discussed. The topics discussed covered the domestic LM PFC design activity for the FNSF. Additional R\&D questions and studies include the following:

- producing a uniform or as nearly uniform as possible liquid Li flowing layer,

- clarifying the Li PFC response to plasma (D and He/Ne fluxes) in combination with $\mathrm{Li}$ and $\mathrm{He}$,

- quantifying vapor shielding and its dependences,

- examining the effect of $\mathrm{H}$ on $\mathrm{Li}$ vapor development and plasma exhaust,

- examining the effects of sheath geometry on impingement and the release of neutral particles and redeposition, and

- investigating the differences between linear and tokamak plasmas.

\subsection{RECOMMENDATIONS AND COMMENTS ON DIAGNOSTICS NEEDS}

These LM studies require a broad set of MPEX diagnostics for the Li PFC performance and plasma response, including impurity spectroscopy, bolometry, and parallel plasma temperature gradients. Some of these are already intended in the MPEX diagnostics plan. However, some of the diagnostics required for these experiments might best be implemented for specific experiments by the LM experiment proponents in collaboration with the MPEX team.

\subsection{RECOMMENDATIONS AND COMMENTS ON PRIORITIES OF MATERIAL SYSTEMS TO BE TESTED}

The recommendations from the community are almost exclusively for liquid Li PFCs to be tested. This is mainly due to the United States's historical leadership in that area coupled with the European Union's focus on liquid $\mathrm{Sn}$.

\subsection{RECOMMENDATIONS AND COMMENTS ON THE TIME LINE AND PRIORITIES FOR MPEX HIGH-LEVEL COMMISSIONING}

No direct recommendations were made regarding the time line and priorities for the MPEX high-level commissioning other than that the use case for liquid Li metal targets and its related inventory should be more closely be assessed. The community decided that the in-vessel inventory of 3.45 mol alkaline metal might be too stringent. 


\section{FUSION ENERGY INDUSTRY PERSPECTIVES}

Fusion industry representatives from Tokamak Energy (TE), Commonwealth Fusion Systems (CFS), and TAE Systems expressed interest in using MPEX for their development paths.

The aggressive time lines of fusion industry do not allow for the development of exotic new PFMs or PFCs as the first choice of PFCs. TE and CFS plan to use high-Z (W) metal walls as baseline PFM choices. TE might consider slow-flowing liquid Li metal divertor PFCs at a later stage of their development path. TAE Systems does not have a material system identified and will leverage mainstream fusion research. The plasma exposure of neutron-preirradiated materials is interesting to fusion industry. TE and CFS divertor designs are standard, and standard mainstream divertor plasma parameters are expected. Thus, MPEX and its capabilities are relevant for testing PFMs and PFCs for the fusion industry representatives from TE and CFS. The requirements for TAE Systems are different and might require changes and upgrades to the MPEX device at a later stage (e.g., biasing of the target).

Important research topics include:

- the importance of PMI chemistry, including wall conditioning;

- H retention in codeposited layers; and

- erosion rate/dust production rate as a function of plasma parameters in divertor.

The fusion industry's aggressive time line in its development path creates an urgency for MPEX exploitation. The industry values a fast, high-level commissioning phase and early long-pulse operation. 


\section{APPENDIX A. AGENDA FOR MURF}

Monday, September 13, 2021

\begin{tabular}{|c|c|c|c|}
\hline Time & Item & Chair/speaker & Min \\
\hline 10:00 & Welcome & Wade & 10 \\
\hline $10: 10$ & Introduction from FES & Clark & $5+5$ \\
\hline $10: 20$ & Introduction to MPEX & Rapp & $25+5$ \\
\hline $10: 50$ & MPEX diagnostics & Biewer & $15+5$ \\
\hline \multirow[t]{2}{*}{ 11:10 } & MPEX high-level commissioning & Rapp & $10+5$ \\
\hline & PMI introduction & Katoh & \\
\hline $11: 25$ & PMI research needs & Wirth & $25+5$ \\
\hline $11: 55$ & PMI research on linear devices in era of fusion nuclear science & Tynan & $25+5$ \\
\hline \multirow[t]{2}{*}{$12: 25$} & Lunch break & & 55 \\
\hline & PMI thrust erosion/redeposition & Abrams/Unterberg & \\
\hline $13: 20$ & $\begin{array}{l}\text { Erosion/redeposition gaps/needs emphasizing appropriate linear-toroidal } \\
\text { device coupling }\end{array}$ & Abrams & 15 \\
\hline $13: 35$ & Implanted depth marker studies for erosion/redeposition & Woller & $10+5$ \\
\hline $13: 50$ & In situ erosion measurement validation efforts/options & Johnson & $10+5$ \\
\hline 14:05 & Erosion properties of advanced $\mathrm{W}$ alloys & Kolasinski & $10+5$ \\
\hline $14: 20$ & Net erosion model validation needs & Guterl & $15+5$ \\
\hline $14: 40$ & $\begin{array}{l}\text { Sheath effects on erosion emphasizing measurement needs for model } \\
\text { validation }\end{array}$ & Curreli & $15+5$ \\
\hline 15:00 & Discussion & Abrams/Unterberg & 30 \\
\hline \multirow[t]{2}{*}{$15: 30$} & Coffee break & & 15 \\
\hline & PMI thrust Solids and Surface evolution & Parish/Baldwin & \\
\hline $15: 45$ & Overview of surface modification experiments and future perspective & Baldwin & $20+5$ \\
\hline $16: 10$ & W fuzz precursors and evolution & Parish & $5+5$ \\
\hline $16: 20$ & Code validation experiments & Dasgupta & $10+5$ \\
\hline $16: 35$ & Changes to thermal conductivity & Simmonds & $10+5$ \\
\hline $16: 50$ & Crack formation in refractory metals due to thermal-mechanical stresses & Ghoniem & $10+5$ \\
\hline $17: 05$ & $\begin{array}{l}\text { Other refractory material irradiation response and possible PMI } \\
\text { relevance }\end{array}$ & Bhattacharya & $10+5$ \\
\hline $17: 20$ & $\mathrm{SiC}$ as plasma-facing material & Sinclair & $10+5$ \\
\hline $17: 35$ & Discussion & Parish/Baldwin & 30 \\
\hline $18: 05$ & Adjourn & & \\
\hline
\end{tabular}


Tuesday, September 14, 2021

\begin{tabular}{|l|l|l|r|}
\hline Time & Item & Chair/speaker & Min \\
\hline & Fusion industry perspectives & Rapp & \\
\hline $10: 00$ & TE & Gryzanovich & $15+5$ \\
\hline $10: 20$ & CFS & Reinke & $15+5$ \\
\hline $10: 40$ & TAE systems & Marshall & $15+5$ \\
\hline $11: 00$ & Discussion & Rapp & 20 \\
\hline & & & \\
\hline $11: 20$ & PMI thrust H retention & Shimada & \\
\hline $11: 30$ & H redention in high-energy ion damaged W & Shimada & 10 \\
\hline $11: 45$ & H retention modeling & Simmonds & $10+5$ \\
\hline & & Marian & $10+5$ \\
\hline $12: 00$ & Lunch break & & \\
\hline & & & 60 \\
\hline $13: 00$ & H retention in neutron-irradiated W & & \\
\hline $13: 15$ & Interpretation of thermal desorption results of H and He in W & Shimada & $10+5$ \\
\hline $13: 30$ & H transport in PFCs and blankets & Zhong & $10+5$ \\
\hline $13: 45$ & Discussion & Humrickhouse & $10+5$ \\
\hline & & Shimada & 30 \\
\hline $14: 15$ & Coffee break & & \\
\hline & & & 15 \\
\hline & PMI thrust LMs & & \\
\hline $14: 30$ & Introduction and high-level vision & Maingi/Allain & \\
\hline $14: 50$ & Needs for flowing systems & Maingi & $15+5$ \\
\hline $15: 05$ & Needs for stagnant/creeping systems & Andruczyk & $10+5$ \\
\hline $15: 20$ & Needs for modeling validation of the plasma response to LM & Goldston & $10+5$ \\
\hline $15: 35$ & Integration aspects with reactor designs & Lore & $10+5$ \\
\hline $15: 55$ & Discussion & Kessel & $15+5$ \\
\hline & & Maingi/Allain & 30 \\
\hline $16: 25$ & Coffee break & & \\
\hline & & & 15 \\
\hline $16: 40$ & Prioritization of research questions, wrap up information & \\
\hline $18: 10$ & Adjourn & Rapp & 90 \\
\hline & & & \\
\hline & & & \\
\hline & & & \\
\hline & & & \\
\hline & & & \\
\hline & & & \\
\hline & & & \\
\hline
\end{tabular}

\section{Participants}

Abrams, Akiyama, Allain, Andruczyk, Baldwin, Beers, Bhattacharya, Biewer, Caughman, Chang, Clark, Collins, Coriton, Curreli, Dasgupta, Doerner, Donovan, Drobny, Ennis, Garrison, Ghoniem, Gietl, Goldston, Gray, Gryhzanevich, Guterl, Hijazi, Humrickhouse, Johnson, Katoh, Kessel, Khodak, Kolasinski, Lau, Lore, Maingi, Marian, Marshall, Nishijima, Parish, Patino, Rapp, Reid, Reinke, Sabau, Scime, Segal, Shimada, Showers, Simmonds, Sinclair, Sorensen, Stangeby, Trask, Tynan, Unterberg, Wade, Wirth, Woller, Zaloznik, Zarkadoula, and Zhong from the following national laboratories, universities, ORNL, and fusion industry:

- University of Tennessee, Knoxville

- Pacific Northwest National Laboratory

- General Atomics

- Massachusetts Institute of Technology

- Idaho National Laboratory 
- University of California San Diego

- University of California, Los Angeles

- University of Illinois Urbana Champaign

- Pennsylvania State University

- West Virginia University

- Auburn University

- TE

- CFS

- TAE systems

- Lockheed Martin 
